\title{
INTRODUCTION TO THE ISSUE
}

When I entered the field of psychiatric rehabilitation ten years ago, I was surprised and pleased to find a clientele with an incredible range of vocational motivation, experience, and potential. Conversely, I was saddened by the lack of effective vocational rehabilitation services for people with psychiatric disability.

What I found instead was a mental health system that considered work to be peripheral to the lives of its clients. At best, work was viewed as a potential therapeutic tool; at worst, work was seen as potentially destabilizing and harmful. Also, I found that traditional vocational rehabilitation service providers, trapped by rigid operational procedures designed to meet the needs of people with physical disabilities, were wary of the heavy investment of time and resources required for assisting people with psychiatric disabilities to select, secure, and sustain employment.

The apparent diminished vocational capacity of the client, the unpredictability of the illness, and the unresponsiveness of mental health professionals frequently combined to result in the denial of much-needed vocational rehabilitation services for people with psychiatric disabilities. In spite of this dismal picture, the families of people with mental illness and the people themselves continued to express a desire to work, and to do work that is both satisfying and meaningful.

Ten years later, my first-hand experiences in providing vocational rehabilitation services to people with psychiatric disability as well as my many encounters with dedicated and innovative professionals in the mental health and vocational rehabilitation services systems, have given me a renewed vision of vocational possibilities for the population.

I am hopeful because I have personally experienced the satisfaction of watching people grow and change vocationally when programs include the correct personnel, program, and system ingredients. I feel expectant because I see consumer and family groups organizing and developing the confidence, competence, and credibility needed to influence decision makers and funding sources to restructure or replace existing programs with those that contribute to vocational outcomes for service recipients. I feel cautiously optimistic because I sense an awakening awareness in both professional communities-mental health and vocational rehabilitation - for the need to relinquish ineffective rituals and to replace them with technologies that achieve the vocational outcomes desired by consumers and their families.

With families, consumers, professionals, and constituents aligned, we must rally to improve our vocational rehabilitation services so that no person with a psychiatric disability who wants meaningful work will be denied that basic human experience. The articles in this issue are an indication that rehabilitation researchers, providers, and educators are doing much to further develop the emerging field of vocational rehabilitation for people with psychiatric disability. The articles reflect an emerging energy in the field of vocational rehabilitation for people with psychiatric disability, and they validate my current feelings of hope, expectancy, and cautious optimism about "the state of the art."

Dr. Gary Bond's update of outcome studies illustrates both the promise of successful programs and the great need for more and better research. Dr. Sally Rogers and her associates have contributed a new research study. Although not an experimental study, the data give evidence that vocational success can be enhanced by participating in psychosocial rehabilitation programs that design programming to encourage and actualize vocational goals of clients.

One of the newest vocational rehabilitation ventures is supported employment. Originally conceptualized for people with mental retardation, supported employment is rapidly becoming a methodology of choice for people with psychiatric disability. However, little outcome research has been available for this population. In their timely article, MacDonald-Wilson, Revell, and associates present a comprehensive description and comparative analysis of both services and outcome for people with psychiatric disability as compared to people with mental retardation. 
Good interagency relationships between mental health agencies and vocational rehabilitation agencies are essential for comprehensive vocational rehabilitation services. The article by Dr. Linda Katz provides us with a view of how these relationships can be enhanced when practitioners from these agencies are trained jointly to provide qualitative services.

Dr. Dennis McCrory, a long-time psychiatric consultant to the Massachusetts Rehabilitation Commission, focuses his attention on the need for acknowledging the importance and power of the "rehabilitation alliance," that is, the mutually respectful relationship that must be the core of the rehabilitation effort. He reminds us that, in the final analysis, the people, not the system, ensure a successful rehabilitation outcome. A look to the future in the article by Dr. Joseph Campbell reminds us of the emerging consumer movement and the need for responsive and inclusive vocational services and programming.

As guest editor for this special issue, I am hopeful that the readers will find its contents as useful and stimulating as they were for me. The vocational needs of people with psychiatric disabilities can and will be met by those professionals who continue to replicate services such as those documented by the contributing authors.

Karen S. Danley, Ph.D. Issue Editor 